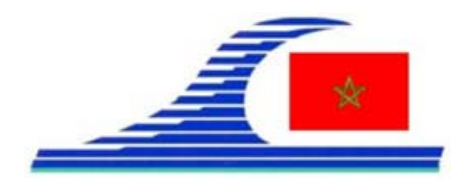

Conférence Méditerranéenne Côtière et Maritime

EDITION 2, TANGER, MAROC (2011)

Coastal and Maritime Mediterranean Conference

Disponible en ligne - http://www.paralia.fr - Available online

\title{
Aspect et répartition de la patelle commune méditerranéenne (Patella caerulea) de la zone côtière oranaise (littoral algérien occidental)
}

\author{
Mohamed El Mustapha KALLOUCHE ${ }^{1}$, Djilali BOURAS ${ }^{1}$, \\ Mohcine GHALEK ${ }^{1}$, Fouad ABDELGHANI ${ }^{1}$
}

\author{
1. Département Biologie, Faculté des Sciences, Université d’Oran Es Senia, Algérie. \\ mediterranoran@yahoo.fr
}

\section{Résumé :}

La présente étude est basée sur l'analyse biométrique de 952 individus du Mollusque Gastéropode Patella caerulea (la patelle commune de la Méditerranée) examinés le long de deux transects de $200 \mathrm{~m}$ de chaque site, qui sont respectivement Ain Defla-Kristel et la digue du port d'Oran.

La distribution et l'évolution des peuplements déterminés sur la base de l'équilibre/déséquilibre d'espèces montre à l'évidence deux systèmes nettement différents: Le premier est impacté (pollué), le second est relativement sain. De ce fait, la patelle commune de Méditerranée (Patella caerulea) affiche une population plus dense et une distribution homogène, au niveau du secteur côtier de la digue du port d'Oran par rapport à celui d’Ain Defla.

Mots clés :

Patella caerulea - Peuplements - Equilibre - Distribution

\section{Introduction}

Le littoral oranais, qui constitue la partie Ouest de la côte algérienne, est caractérisé par de multiples phénomènes de diverses origines, qui ont de fortes empreintes écologiques. C'est un environnement dont l'équilibre dépend des facteurs biotique (biodiversité méditerranéenne), abiotique (hydrodynamisme et changements climatiques), et surtout anthropiques (croissance démographique, invasion du littoral, rejets urbains et industriels) (BOURAS, 2007). A cela, s’ajoute un mouvement de marée quasi nul mis.

Le choix de l'espèce Patella caerulea revient à son abondance dans la région oranaise, les biotopes où elle évolue sont accessibles. De ce fait, on s'est basé sur l'analyse biométrique de 952 individus de Patella caerulea dans les deux secteurs côtiers : Ain Defla (Kristel) et celui de la digue du port d'Oran, dans le but de comprendre et suivre les différents comportements et réponses de Patella caerulea par rapport aux différentes interactions environnementales et écologiques.

Secteur côtier d'Ain Defla-Kristel (3550,761' N et $00^{\circ} 29,223^{\prime}$ W) : Cette portion côtière rocheuse est composée de plusieurs structures (galets, sables, plates formes à vermet, microfalaises...). Seules les microfalaises et les plates-formes à vermets on été choisit 
La connaissance de la Mer :

un vecteur du développement durable en Méditerranée

étant donné qu'elles constituent un habitat favorable pour la survie les différentes espèces de patelles. Notons que ce site est relativement éloigné de toute pression urbaine ou industrielle.

Secteur côtier de la digue port d'Oran $\left(35^{\circ} 42,811^{\prime} \mathrm{N}\right.$ et $\left.00^{\circ} 39,359^{\prime} \mathrm{W}\right)$ : Le secteur du Port d'Oran constitue le premier port du secteur Ouest de l'Algérie. La jetée de la digue de ce port est constituée de brise-lames cubiques faits de béton armé.

\section{Matériel et méthodes}

L'étude a été menée dans toutes les conditions météorologiques et en se déplaçant à pied sur l'estran selon une méthode non destructive.

Un tracé de 20 mètres est établi sur l'estran (la ligne de rivage ; long-shore) à l'aide d'un décamètre (figure 1). Trois quadrats de $1 \mathrm{~m}^{2}$ (une surface de $1 \mathrm{~m}^{2}$ délimitée par un cadre en bois ou en PVC) sont posés aléatoirement sur ces tracés au niveau de l'estran. Les patelles qui se trouvent au niveau de ces quadrats sont comptés puis certains paramètres biométriques sont prélevés (figure 2). Ensuite elles seront remises à leurs places, pour que le stresse induit sur elles soit le moins perturbant possible. Cette opération est répétée plusieurs fois en allant vers l'est de façon à couvrir une distance totale de 200 m de côte sur chaque site.

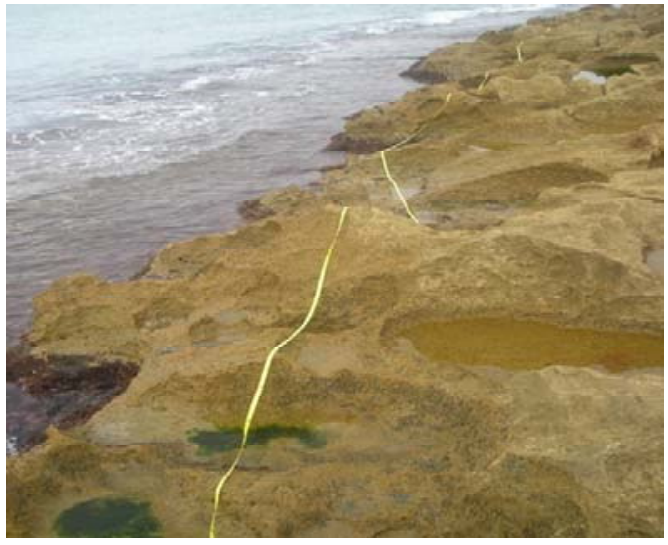

Figure 1. Tracé de 20 m à l'aide du décamètre à Ain Defla-Kristel.

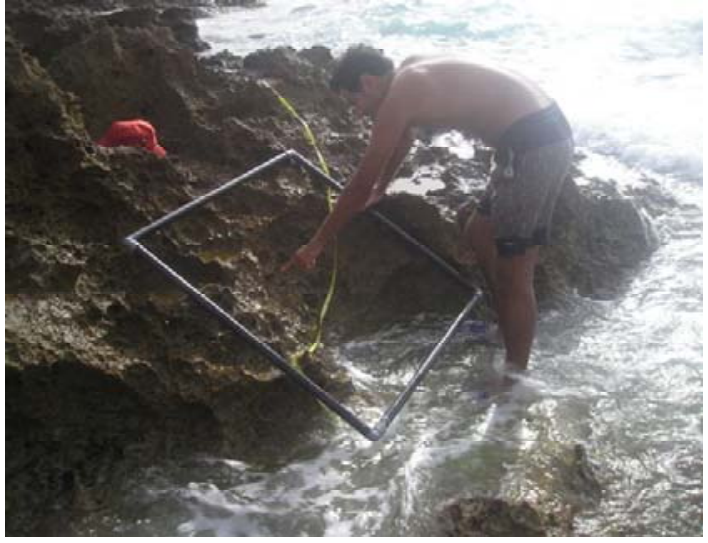

Figure 2. Quadrat de $1 \mathrm{~m}^{2}$ à Ain Defla-Kristel.

Ces paramètres biométriques servent à l'application des statistiques sur la population de patelles vivantes. Pour les relations d'allométrie et de la croissance relative, nous avons effectué les trois mesures suivantes sur les 952 individus de Patella caerulea échantillonnés sur les $200 \mathrm{~m}$ de chaque site :

- Longueur antéropostérieure de la coquille (L) ;

- Hauteur de la coquille (l'épaisseur) $(\mathrm{H})$;

- Poids (P) : le poids frais total (chair et coquille) de chaque individu a été mesuré. 


\section{Résultats et discussion}

\subsection{Croissance linéaire}

Toutes les relations biométriques effectuées sur les individus de Patella caerulea, prélevés à Ain Defla-Kristel montre une corrélation bien supérieure à ceux prélevées au niveau de la digue du port d'Oran. Ces corrélations nous indiquent que les patelles du secteur côtier d'Ain Defla-Kristel, sont plus en équilibre biologique par rapport à ceux de la digue du port car plus le coefficient de détermination $\mathrm{R}^{2}$ se rapproche de 1 plus la corrélation est bonne et plus il est en équilibre biologique. En effet, on constate que le coefficient de détermination $\mathrm{R}^{2}$ de la population des Patella caerulea du port d'Oran est nettement infèrieure à celui d’'Ain Defla-Kristel.
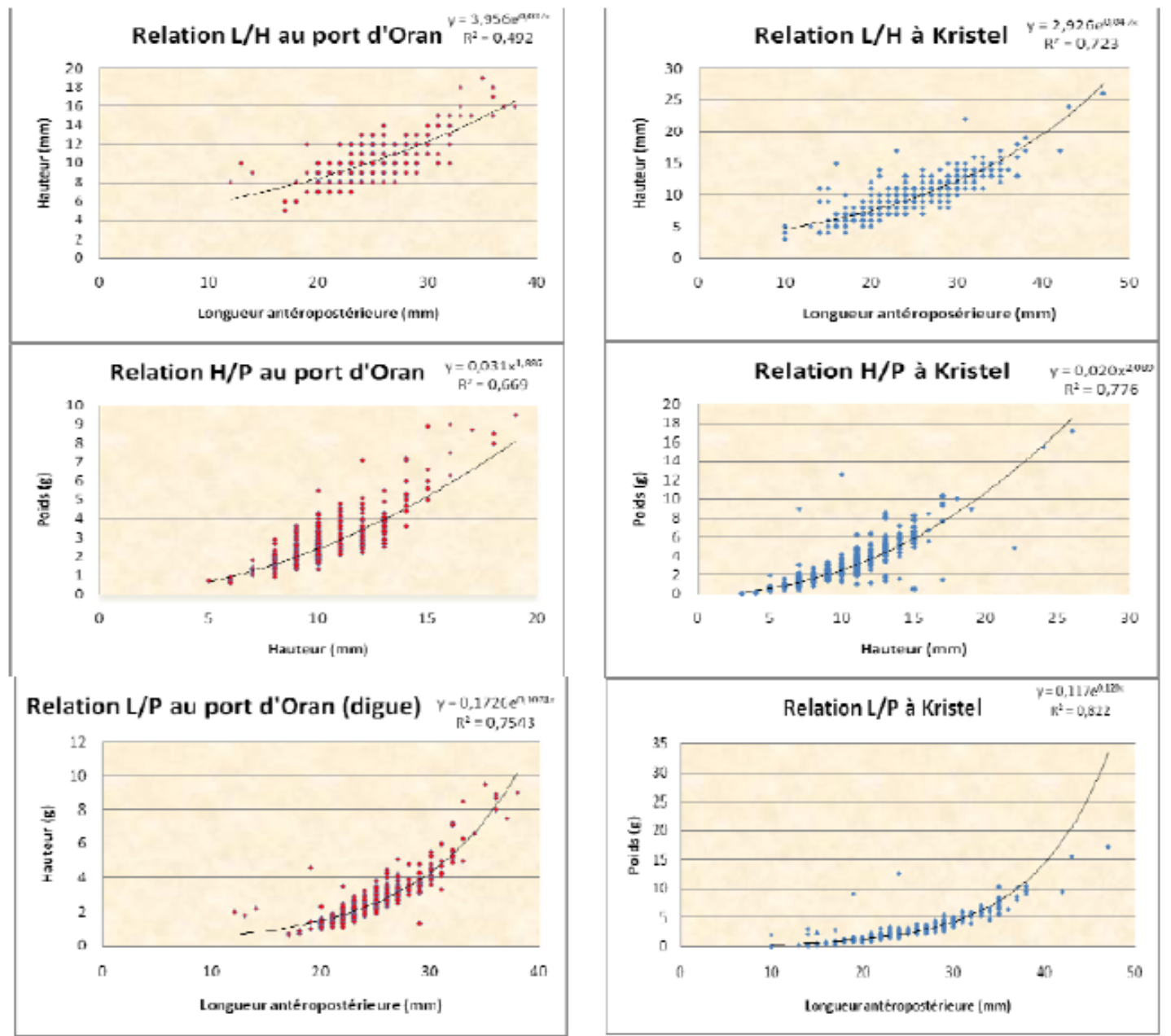

Figure 3. Relation entre le poids et la hauteur des individus Patella caerulea.

\subsection{Distribution des classes de taille}

On note en premier lieu, le nombre de classes de taille d'Ain Defla-Kristel qui comporte 8 classes de taille, alors qu'il y a 6 classes de taille au niveau de la digue du port d'Oran. 
La connaissance de la Mer :

un vecteur du développement durable en Méditerranée
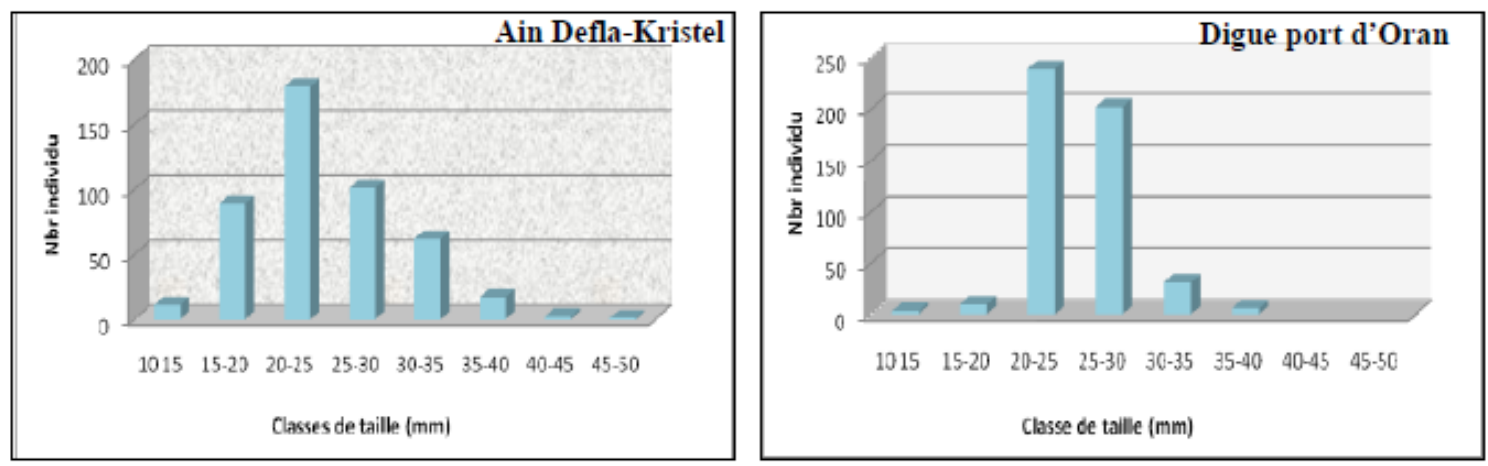

Figure 4. Les classes de taille de Patella caerulea des deux sites.

L'ensemble de la classe de taille comprise entre 10-15 mm compte 14 individus, 78.5\% à Ain Defla-Kristel. Pour la classe de taille de 15-20 mm, on dénombre 99 individus sur la totalité des deux sites, $89.9 \%$ pour Ain Defla et $10.1 \%$ au port. Sur un total de 418 individus de la classe de taille de $20-25 \mathrm{~mm}, 57.2 \%$ appartiennent aux individus prélevés au port. Sur 303 individus de la classe de taille de $25-30 \mathrm{~mm}$, les deux tiers se trouvent au niveau du port $66.7 \%$ et un tiers à Ain Defla. Cependant, on constate l'inverse pour la classe de taille 30-35 mm qui regroupe au total 93 individus. Une nette dominance a été enregistrée sur les patelles échantillonnées à Ain Defla. En effet, sur les 23 individus de la classe de taille de $35-40 \mathrm{~mm}$, il n'y a que $26 \%$ qui représentent le port d'Oran. Pour les deux classes suivantes $40-45 \mathrm{~mm}$ et $45-50 \mathrm{~mm}$, on ne dénombre que trois individus qui ne se localisent qu'au niveau d'Ain Defla.

L'intervalle de confiance permet de constater que la différence est significative entre la même classe de taille des deux sites, car l'Ic est toujours superieur à 1,96.

On constate une abondance des individus de très petite taille et de grande taille (supérieure à $30 \mathrm{~mm}$ ), au niveau d'Ain Defla-Kristel. Cependant, les individus de taille moyenne (entre 20 et $30 \mathrm{~mm}$ ) sont importants au port d'Oran en nombre par rapport à ceux d'Ain Defla-Kristel même au-delà des transects de l'étude.

Le test de conformité est significatif, cela indique la différence des répartitions des classes de taille (théoriques et observées) sur les deux secteurs côtiers.

\subsection{Distribution de la densité}

On remarques une stabilité de la moyenne du nombre des individus de Patella caerulea par $\mathrm{m}^{2}$ sur les 200m de la digue du port d’Oran (coté de la jetée). En revanche, ce nombre varie de 7 à 55 individus de moyenne par $\mathrm{m}^{2}$ par tracé sur les 180 premiers mètres étudiées à Ain Defla, au delà de cette distance, une diminution de populations de patelles a été enregistrée. En effet, une brusque diminution a été observée au niveau des quadrats allant de 55 à 19 individus jusqu'à la totale disparition arrivant à $200 \mathrm{~m}$. Ce constat met à l'évidence que la densité des individus change avec la morphologie du substrat qui l’héberge. 


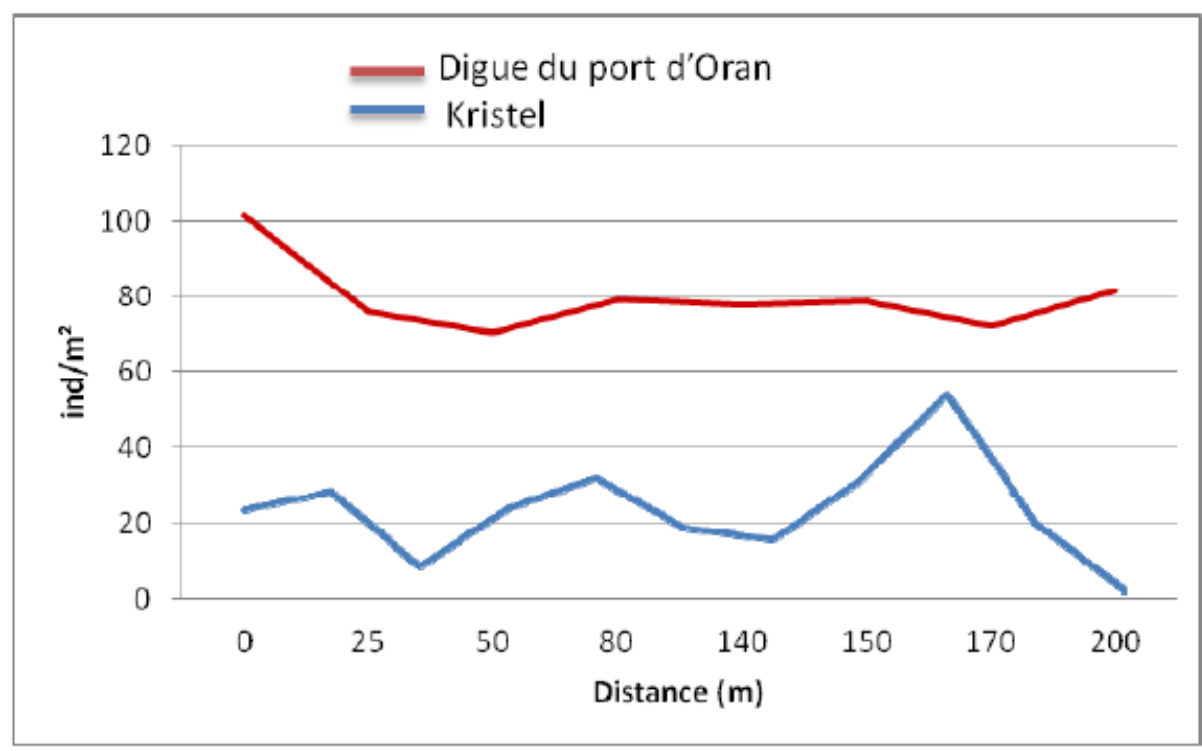

Figure 5. Distribution moyenne par $m^{2}$ du nombre d'individus sur une distance de 200 m de côte au niveau d'Ain Defla-Kristel et la digue du port d'Oran.

Le nombre d'individu de Patella caerulea au port d'Oran est nettement superieur à celui de Kristel. En effet, ce nombre est d'environ 80 individus de moyenne par $\mathrm{m}^{2}$. Alors qu'il n'atteint qu'une moyenne de 25 individus par $\mathrm{m}^{2}$ à Kristel. Cette différence entre ces valeurs est significative, car le test de significativité $\mathrm{G}$ est de 4.38 , donc supérieur à 1.96 confirmant la différence des résultats

\subsection{Distribution de la biomasse}

La biomasse totale de la population de Patella caerulea, de la digue du port d'Oran, dépasse largement celle d'Ain Defla-Kristel. La moyenne de la biomasse totale au niveau du port est de $227 \mathrm{~g} / \mathrm{m}^{2}$. Tandis que, cette biomasse est de $67 \mathrm{~g} / \mathrm{m}^{2}$ au niveau de Kristel.

Le pique de la population de Patella caerulea, enregistrée à Ain Defla (jusqu'à 70 individus $/ \mathrm{m}^{2}$ ), est situé dans une zone où les vagues frappent de plein fouet la côte (VELA \& LEONI, 2004). Ce qui explique aussi cette forte densité régulière et homogène de patelles au port d'Oran, car l'eau est bien oxygéné en raison du renouvellement des eaux marines fortement secouées par un hydrodynamisme local intense.

\section{Conclusion}

La patelle commune méditerranéenne Patella caerulea n'est très dense qu'au niveau des secteurs côtiers faiblement fréquentés. Son abondance est due à sa grande fertilité, sa faible cueillette, sa résistance face à la pollution et à la morphologie du substrat dur qui l'héberge. En effet, au niveau de la digue du port d'Oran, où l'activité humaine est 
La connaissance de la Mer :

un vecteur du développement durable en Méditerranée

limitée vu l'accès interdit dans cette zone, la population de la patelle est très abondantes par rapport au secteur côtier d'Ain Defla-Kristel. Cependant, les différents rapports obtenues à partir des données allométriques montrent qu'il y a une plus forte corrélation des patelles à Ain Defla-Kristel par rapport à celles du port d'Oran.

La physiologie des organismes (croissance, reproduction) est étroitement liée à la quantité de nourriture disponible. La nutrition a un effet direct sur la vitesse et la durée des phénomènes de gamétogenèse (LUBET, 1980 in MISSILI, 2002). Cet état est sans doute la cause de la plus grande abondance de patelles au port par rapport à Kristel. En effet, l'émissaire, d'eau usée, de fort Lamoun alimente la digue en matière organique qui favorise la multiplication des microalgues qui sont la base de nourriture des patelles. La couverture algale et la composition floristique jouent un rôle prépondérant pour l'abondance des herbivores (HEREU, 2004).

Au vu de la répartition des classes de taille, Patella caerulea au niveau d'Ain DeflaKristel, évolue en harmonie dans cet écosystème qui reste encore à l'état sauvage. Néanmoins, on note qu'à la digue du port d'Oran (nombre d'individus et biomasse élevée, absence de certaines classes de taille) pourrait être lié à la l'importante fertilité de l'espèce, l'alimentation abondante et vraisemblablement à la durée de vie plus réduite à cause de la pollution qui se trouve être un grand facteur de stresse.

\section{Références bibliographiques}

BOURAS D. (2007). Dynamique bioclimatique et morphologique de la zone côtière oranaise : approche éco-biologique (Algérie nord occidentale). Thèse doctorat, Univ. Es Sénia, Oran, Algérie, 200 p.

HEREU B. (2004). The role of trophic interactions between fishes, sea urchins and algae in the northwest Mediterranean rocky infralittoral. Thèse Doctorat, Univ. Barcelona, 287 p.

MISSILI A. (2002). Ecologie de la moule Mytilus galloprovincialis de la baie d'Alger. Thèse Magister, Univ. USBH Alger, Algérie, 97 p.

VELA A., LEONI V. (2004). Etudes des espèces de l'étage médiolittoral sur la jetée du port de commerce de Bastia. Recensement des effectives de Patella ferruginea. Contrat Sintinelle \& Collectivité Territoriale de Corse, France, 18 p. 